\title{
9. REGISTER
}

\subsection{Personenregister}

Da Guibert de Nogent auf nahezu jeder Seite genannt wird, wurde auf die Aufnahme seines Namens in das Personenregister verzichtet. Hochgestellte Zahlen nach den Seitenangaben beziehen sich auf die Anmerkungen.

Aaron 44,49

Abaelard $81^{221}, 84^{244}, 216^{69}$

Abbo, Abt von Fleury $131^{+189}, 133$, 136f., 157

Adam 44

Ademar von Chabannes $21,68^{169}, 83^{+237}$, $134^{204}$

Adgar 229

Adrian, Hl. $\quad 213^{53}, 256$

Agatha, $\mathrm{Hl} . \quad 213^{53}, 256$

Agnes, $\mathrm{Hl} . \quad 213^{53}, 256$

Alfegus, Erzbischof von Canterbury 39, $40^{18}, 66,72,86,94$

Alard, Abt von Florennes $25^{118}, 26,220$

Aldhelm, Confessor 86

Alexander III., Papst 66

Alexander Comnenus, Kaiser $68^{169}$

Alexander der Große, König 49-52, $56^{+110}, 57,106,126$

Alfred, König $131^{188}$

Alkuin $59^{124}$

Alphons der Weise 230

Alvisius, Bischof von Arras 220

Amandus, Abt von Marchiennes 221

Amatus von Oloron, Legat 88

Ambrosius Autpertus 129180

Andreas, Abt von Nogent 27

Andreas von Saint-Victor $51^{86}$

Angilbert, HI. 72, $90^{276}$

Anscherius, Abt von Saint-Riquier 72

Ansellus, Dieb $163 \mathrm{f}$.

Ansellus, Matricularius $154^{303}$

Ansellus, Sakristan 125

Anselm von Bec, Erzbischof von Canterbury, $\mathrm{HI}$. 3, $4^{13 f}, 19,39 \mathrm{f}$., $44,47^{62}, 51$, $66,72^{+183}, 81,86^{257}, 93 \mathrm{f} ., 109,111-114$, $116^{+111}, 117$ f., $122,136,138,142,158^{316}$, $176,232,233^{136}, 248 \mathrm{f} ., 253$

Anselm von Chiusa $232^{129}$

Anselm von Laon $3,24,123 \mathrm{f}$.

Anselm von Lucca 86

Anselm von Ribemont $120^{131}, 236^{160}$

Anselm von Saint-Rémi $47^{61}, 205^{21}$
Anselm von St. Saba 117,138

Aristoteles $95^{5}$

Arius $118^{117}$

Arnulf von Crépy, HI. $147^{266}, 152$, $157^{+309}, 172,179$

Arnulf von Soissons, $\mathrm{Hl} . \quad 25^{114}, 80^{+212}, 84$, $88^{+262}-91,119^{+122}, 122^{136}, 158,205^{20}, 248$

Artaldus, Erzbischof von Reims 54, $154^{303}$

Astronomus $62^{139}$

Aubertus, Kleriker 212, 223

Audifax, Hl. 204

Augustinus, $\mathrm{Hl}$. 29f., $39^{13}, 40^{+24}, 43 \mathrm{f}$., $45^{43}, 48,50^{+57 f}, 51,57^{116}, 61,62^{138}, 67$, $76^{197}, 84,93,102^{44}, 166,189^{120}, 194^{147}$

Balduin, König 120

Balduin von Edessa 160, 196

Baldwin, Abt von Bury St. Edmunds 133, 137

Bandaridus, Hl. $217^{77}$

Barthélemy de Jur, Bischof von Laon $14^{52}, 23^{109}, 119-121,157,234^{145}, 238 \mathrm{f}$.

Benedikt, HI. 117, 157, 171

Benedikt IX., Papst 91

Bernard, Dekan von Soissons 24

Bernhard, Abt von Signy $237^{160}$

Bertram, Archidiakon 133

Bertrand de Comminges, Hl. 111

Bileam 44, 53, 57+115, 58, 200

Boso, Abt von Saint-Benoît-sur-Loire 212

Brioccus, Hl. 208

Bruno der Kartäuser 162

Burchard, Bischof von Worms 85

Caesar, Gaius Julius $\quad 52,98^{17}, 99$

Calixt II., Papst $87^{+220}, 90,220$

Cassiodor $51^{83}$

Catarina, Hl. $213^{53}, 256$

Celsus, Hl. 91

Chlothar, König 223

Clarembald von Roset 237160 
Clarius von Sens $88^{262}, 91$

Claudius von Turin 38

Clemens, Hl. $213^{53}, 256$

Clemens III., Papst 117

Clement von Bucy $84^{+244}$.

Constanze, Königin $\quad 209,212,216,218 f$., 222,224

Cornelius, Hl. $80,157,170$

Cyprianus von Karthago, $60^{+127}, 157,170$

Dalmatius von Semur 115

Daniel 51

Desiderius von Montecassino 116-118, $171^{43}, 175$

Dionysius, Hl. $\quad$ 157, 172, 179, 186, 212

Dominic von Evesham 232

Drogo, Bischof von Beauvais $154^{302}$

Dungal von Saint-Denis $38^{+9}$

Eadmer von Canterbury $20 \mathrm{f} ., 40^{+18}$, $72^{183}, 116,134^{204}, 248$

Edmund, König, Hl. $\quad 46,131-138,152$, $157,172,179-181$

Eduard der Bekenner, König 63, 133, 137

Eleutherius, Hl. $\quad 212^{48}$

Elija 217

Embricho von Mainz $194^{149}$

Erlebald, Dekan 61

Erlebaldus, Büßer $\quad 77^{+201}$, 119f., 151, 154, 157

Ernulf, Mönch $113^{89}$

Etienne de Garlande $80^{213}, 121^{135}$

Exuperius 68

Eugen II., Papst 203, 220

Eusebius von Caesarea $51,118^{117}$

Eva 44

Evrard von Breteuil $\quad 163^{7}$

Evrard von Bucy $84^{244}, 162$

Firminus, HI. $\quad 26,27^{+131}, 68,121,186^{107}$

Flavius Josephus 50f., 56, 126

Flodoard von Reims $145,148^{272}, 229$

Fulcanus, Dekan $212^{+51}, 222$

Fulcher von Chartres $33,131^{190}, 141^{+237}$, 159f., 192f., 195-197+163

Fulchoinus s. Fulcanus, Dekan

Fulchoisus s. Fulco, Bischof von Soissons

Fulchosius s. Fulco, Bischof von Soissons

Fulco, Bischof von Soissons $212^{+49}, 216$, 218

Fulcuinus s. Fulco, Bischof von Soissons

Fulco, Archidiakon $82^{233}, 211$
Gaiferius von Montecassino $115,116^{105}$

Garnier, Abt von Saint Germer de Fly $113^{90}$

Gaudry, Bischof von Laon 3,25, 119, $124^{151}, 138,165,174$

Gaufrid, Abt von Saint-Médard de Soissons $25^{+119}, 26,218,220^{+82}, 221,223$, 252

Gauthier von Coincy 116, 230

Geoffroy von Monmouth 7

Geoffroi Cou de Cerf s. Gaufrid, Abt von Saint-Médard de Soissons

Gérard von Quierzy, Kastellan 174

Geremarus, H1. 151

Gervasius, Märtyrer 143

Gibbuinus, Mönch 212

Gil de Zamora 235, 244

Gildardus, Hl. 184, 212, 222, 224

Giraldus, Hl. 114

Godefridus, Graf von Namur 100

Goffridus s. Joffredus von Semur

Gonzalo de Berceo 230

Goswin, Mönch 220

Gottfried, Bischof von Amiens, Hl. 26f., $72^{+185}, 80^{214}, 82^{233}, 90^{276}, 119^{+1.22}$, $121^{+135}, 130^{+183}, 186^{107}, 239,241,243$

Gottfried, Bischof von Beauvais $170^{40}$

Gregor I. der Große, Papst, Hl. $\quad 40^{+24}$, $43^{+30}, 45,49^{72}, 51,53 f$., $59^{124}, 61^{137}$, $62^{138}, 69^{172}, 101,104,139^{231}, 181,184$, $189^{120}, 203 \mathrm{f}$., 208-210, 212, 213 ${ }^{53,55}, 214$, 216-220, 222-225, 230 ${ }^{129}, 255-268$

Gregor VII., Papst $88,114^{102}, 117,171^{43}$

Gregor von Tours, Bischof $45^{+47}, 71^{178}$, $76^{197}, 102,104,228^{+115}$

Gualdricus, Bischof von Laon 3,25

Guido von Beauvais $113^{90}$

Guido von Vienne $\quad 87^{260}$

Guillermus, Mönch $24^{110}$

Gundulf 84

Guorlescius von Quimperlé, HI. 87

Guy, Bischof von Beauvais 18,115

Habacuc, HI. 204

Hariulf, Abt von Oudenburg $88,89^{+269}$

Haymo 186

Heinrich II., Kaiser $220^{88}$

Heinrich von Porcien $237^{160}$

Helgaud von Fleury 64, 146

Helinand, Bischof von Laon 138, 233

Hélissende von Chester, Gräfin $108^{+68}$, 109

Henry, Henri I. von Eu, Graf $109^{73}, 236^{156}$ 
Herbertus, cancellarius 206

Heribertus, Graf $210,212,216,218 f$., $222,224^{100}$

Hermann, Archidiakon $\quad 133^{+201}, 134,136$, $137^{+221}, 152$

Hermann von Tournai $13,17,123 \mathrm{f}$, $145^{+258}, 150,164^{12}, 168^{+29}, 189,220^{82}$, $227^{+109}, 233^{+139}, 234^{+142}$

Hieronymus $48,57^{116}, 102^{46}, 232^{129}$

Hilarius, $\mathrm{Hl} . \quad 213^{53}, 256$

Hildefonsus von Toledo $228^{114}, 230^{+129}$

Hildefonsus Vrayet, Mönch $213^{54}$

Hinkmar, Erzbischof von Reims $126^{159}$

Hippolyt, HI. 74, 148, 189, 244

Hirtius Pansa $98^{17}, 99$

Horaz $131^{190}$

Hugo, Graf von Chester 109

Hugo, Mönch 87

Hugo Farsit $146^{258}, 227,269$

Hugo von Beauvais, Diakon $121^{135}$

Hugo von Die, Legat $88,115^{+102}$

Hugo von Flavigny 117

Hugo von Fleury $97^{+13}, 192^{136}$

Hugo von Lyon 117

Hugo von Saint-Victor $115^{104}, 116,139$

Hugo von Semur, Abt von Cluny $87^{+261}$, 114-117

Ingelrann, Archidiakon $82^{233}$

Ingelrann von Coucy 100

Isidor von Sevilla 30,98

Ivo von Chartres $80^{213}, 81,85 f ., 113^{89}$

Ivo, Abt von St. Denis $153^{+296}$

Jacobus, Hl. 114

Jacobus de Voragine 103

Jesus Christus 12, 31, 37-40, 43f., 48 ${ }^{72}$, $52,59,60^{+125}, 65^{154}, 67 \mathrm{f}$., $123,129,144$, $148,174^{49}, 182 \mathrm{f}$., $197,200,202,203^{+7}$, 205f., 209, 210, 212, 223, 228 $+110,247$

Jocelin von Brakelonde 136

Joffredus, Bischof von Chartres 90

Joffredus von Semur $113,115,117$

Johannes, Abt von Saint-Nicaise $153^{+296}$, $236^{159}$

Johannes, Apostel $\quad 33,67^{165}$

Johannes XV., Papst 86

Johannes der Täufer $68^{+169}, 94,199,205$

Johannes von Salisbury $55^{17}, 51^{85}, 52^{+93}$, $56^{110}, 168^{33}$

Johannes von Soissons, Graf $24,152^{+289}$, 163f., $173^{44}, 177$

Johannes, Bischof von Thérouanne $121^{135}$
Jonas von Orléans $38^{+9}$

Joslenus von Vierzy, Bischof von Soissons $207,213,214^{56}, 218,220,224$

Judas Ischariot $43^{31}, 118^{117}$

Justus, Hl. $157,169,170^{+40}$

Kaiphas, Hohepriester $57,58^{+116}, 200$

Karl der Große, Kaiser $\quad 62^{+139}, 134^{204}$, 203, 209, 212, 223

Karl der Kahle, Kaiser $\quad 154^{302}, 204,210$, 212, 216, 218f., 222f., $225^{102}$

Katharina, Hl. s. Catarina

Knut Ericson, König 66

Konstantin, Kaiser 51

Kuno, Kardinalbischof von Praeneste, Legat $80^{214}, 91$

Lambert, Bischof von Arras 71f., 81226, $82^{232}, 121+134 \mathrm{f} ., 122$

Lambert, Bischof von Guînes 81

Lambert, Bischof von Toumai/Noyon 88-90

Lanfranc von Bec/Canterbury, Erzbischof $14,39 f ., 66^{+159}, 72,86,94,113^{+89}$, 248,253

Laurentius, Hl. $\quad 181,212,219,223 f$.

Leo IX., Papst $47^{61}, 80^{219,221}, 205$

Leocadia von Toledo, Hl. 203, $204^{15}$

Leodegar, Hl. $41 \mathrm{f} ., 107,146,157,172,179$

Leodegar, Bischof von Viviers 74f., 119, $121,124,238 \mathrm{f} ., 242$

Leofstan, Abt von Bury St. Edmunds $134,136,141,152$

Leofstan 133

Letald von Micy $112^{84}$

Lietbert, Bischof von Cambrai 21355, $220^{88}$

Lisiard, Archidiakon 154

Lisiard, Bischof von Soissons $25^{+114}, 84$, $89^{+269}, 111^{82}, 158^{+314}, 202^{6}$

Lisiard, Subdiakon $121^{121}$

Losinga, Bischof von East Anglia $137^{221}$

Lucia, Hl. 256

Ludwig, Hl. $87^{258}$

Ludwig der Fromme, Kaiser $\quad 62^{+139}, 183$, 206, 210

Ludwig VI., König $48,62 f .3,75,108,110$, $119,220,238$

Macutus, Hl. 41

Magdalena, HI. 189

Manasses, Bischof von Soissons $142^{241}$, $152,158,162,196$ 
Manasses II., Erzbischof von Reims $120^{+131}$

Marcellinus, Hl. 204

Marcellus, Hl. $130,140,157,169 \mathrm{f}$.

Maria, Hl. 11f.2, 33, 43, 45, 52f., 67, 74, $109,113,124 \mathrm{f} ., 128 \mathrm{f} ., 148,152-154,158$, $163,168-172,177-179,187-190,199 \mathrm{f}$., $210,211^{+44}, 228^{+113}, 230^{129}, 244,247,250$

Maria Egyptica, H1. $211^{44}$

Maria Magdalena, Hl. $211^{44}$

Marius, Hl. 204

Martha, HI. 187, 204

Martialis, $\mathrm{Hl} . \quad 83^{237}$

Mathilde von Tuszien 117

Medardus, $\mathrm{Hl} . \quad$ 184, 203f., 212, 222, 224

Mohammed $194^{+149}, 195^{153}$

Moses $49^{72}, 52^{+91}, 56,57+112 f$. $217+77$

Murieldis 230, 232129

Nicasius, Hl. $124,128,148,157,169,170$

Nicolaus von St. Crépin/Soissons, Mönch $26,27,119^{122}$

Ninian, Hl. $59^{124 f}$.

Nithard $204^{+15}, 210$

Norbert von Xanten $15^{61}, 19,25^{+117}$

Octavian, Kaiser 52

Odilo 204, $225^{102}$

Odo, Abt von Saint-Rémi 236159

Odo, Abt von Saint-Symphorien $\quad 25^{+116}$

Odo, Bischof von Bayeux 68

Odo II., Bischof von Ostia $101^{40}, 115^{102}$, $117+113$

Ordericus Vitalis $20 \mathrm{f} ., 97^{13}, 99,101,118$

Orosius $52^{+94}$

Osbert von Clare 20, 135

Otloh von St. Emmeram 20

Otmar von St. Gallen, Hl. $86^{257}$

Otmund, Mönch 152, 178

Otto, Diakon 105

Otto von Freising 7

Paschalis II., Papst 79

Paschasius Ratpertus $59+124$

Patiens, Bischof von Metz 205

Paulus, Apostel 50,181

Paulus Diaconus 59124

Petrus, Apostel 204

Petrus Calò 235, 244

Petrus Damiani $58^{+117 f}, 67,92$

Petrus Heremita $33^{173}$

Petrus von Grenoble $73-75,88,106,119$, $121,124,148,149^{276}, 150,228,233$,
$235^{+149}, 237^{163}, 238-242,244,246,252$, 269-277

Philibert de la Mare $237^{166}$

Philipp I., König 63, 79, 88

Pierre de Cuisy, Magister $237^{168}$

Piro, HI. $65,66^{+155,157,126}$

Poppo, Bischof von Trier 91

Pons, Abt von Cluny 87

Prothasius, Märtyrer 143

Pyrrus 193f.

Quilius, König von Jerusalem $100^{32}$, $128^{+171}$

Quintilian 98

Radbod, Bischof von Noyon $\quad 128,129^{180}$, $130^{+183}, 178$

Radulf, Erzbischof von Reims $76,89^{+269}$, $90,120 \mathrm{f}$.

Radulf von Laon 24, 124

Raimond d'Aguilers 33, $192^{136}$

Raoul, Abt von Saint-Médard de Soissons 25, 221

Regnaldus, Mönch 256

Remigius, HI. 54, 80

Richard, Richter 171

Richard, Abt von Saint-Médard de Soissons $212,218,222$

Richard von Saint-Vanne $220^{88}$

Robert, Abt von Bury St. Edmunds 138, $221^{96}$

Robert, Archidiakon 123

Robert, Bischof von Arras $121^{+134}, 81$

Robert I. von Flandern $68^{169}$

Robert II., König 63, 146, 209, 215

Roger fiz Wimund $232^{129}$

Rohardus 27

Roscelinus $84^{244}$

Rudolf von Turne 237160

Rufinus $118^{117}, 126^{+159}$

Rusticus, HI. $212^{48}$

Sacharja $191^{131}$

Salomon $23^{107}, 187$

Samson, Abt von Bury St. Edmunds $135 \mathrm{f}$.

Samson, HI. $\quad 66^{+155}, 126$

Sarah 44

Sardanapalus $61^{+131}$

Sebastian, H1. $184,203,204^{+15}$, 208f., $212,214,219,222,224,255-268$

Sigebert von Gembloux $21^{+96}, 89^{269}$

Sigfrid, Abt von Saint-Vincent 24 
Simon von Crépy $157^{309}, 162^{+6}$

Soiburga 150,234

Sueton 52, 126

Swithun, Hl. 157, 172, 179

Symeon von Trier, Hl. 91

Theodeberta 150,234

Theodericus von Saint-Rémi, Hl. 101

Theodosia, Jungfrau, HI. 129180

Theodredus, Bischof 133

Thomas von Marle $80^{215}$

Thomas von Monmouth $101^{40}$

Thiofrid, Abt von Echternach $39^{+11-13}$ f., $44^{34}, 180^{87}$

Tíburtius, Märtyrer 203

Tudebode, Peter 33

Turstan, Mönch 135

Ulrich, Bischof von Augsburg, HI. 86

Urban II., Papst $49,81,82^{232}, 87^{+259}$, $115^{102}, 117^{+113}, 195$

Ursio, Bischof von Soissons $\quad 80^{212}, 88$
Veranus 118, $171 f$.

Vespasian, Kaiser $52 \mathrm{f}$, 200

Victor III., Papst $117^{+115}, 118,171^{+43}$

Vinzenz von Beauvais 235

Virgil, Bischof von Salzburg, Hl. 105

Walter Map 7,10763

Warin, Abt von Malmesbury 86

Wilhelm, Knabe 152

Wilhelm der Eroberer 153

Wilhelm Rufus, König von England 27

Wilhelm von Champeaux, Bischof von

Châlons 75, 119, 121, 238f.

William I., Graf von Eu 108

William V., Graf von Aquitanien $68^{169}$

William von Malmesbury $7,21,63^{+144}$, $64,86,96^{9}, 97^{13}, 116,118^{+118}, 133,135$, 138

Yvo von Saint-Quentin, Mönch 153

Zosimus $11^{44}$ 


\subsection{Ortsregister}

Abingdon $134^{202}, 136$

Ägypten $51^{87}, 217$

Ailette 109

Amiens $26^{+125}, 27^{131}, 68,71 \mathrm{f}$., $82^{+233}, 121$, 239

Anchin 8f., 24, 28, 158+316, 212, 213 +53 , $214,216-220,222,225^{+102}, 226,252,255$ f.

Angers $145^{258}, 186,208^{+30}, 209^{+32}, 211$

Antiochia 192, 193

Apulien 195

Aquitanien $12,68^{169}, 212,223$

Arras $59^{+123}, 81-83,91^{+282}, 121^{+135}, 145$, $208^{30}, 220,234,269$

Artois 61

Aulne $32^{164}, 234^{147}$

Auxerre $170^{40}$

Basel $235^{149}, 237$

Battle Abbey 27

Beaupré $129^{182}, 236$

Beauvais 18f., 24, 25+116, 78, 80+215-217, $85,87,88^{+262}, 90,111^{82}, 113^{+89 f}, 114^{102}$, $115,121^{135}, 137,140,145,153,154^{+302}$, $160,162,170^{40}, 172,196,235,239$

Bec, Le $109^{+72}, 112,113^{+89}, 137$

Beodricsworth s. Bury St. Edmunds

Bourbourg 61,77

Burgund 113

Bury St. Edmunds $117,133,137^{+221}$ f., $152,157,233$

Buzançais $145^{258}$

Caen 145

Cambrai $61,77^{+201}, 81,102^{46}, 129181,145$, $157,213^{+55}, 234,236^{160}, 237,243$

Cambron 238

Canterbury $39,66,72,113^{+89}, 116 f ., 123$, 138

Catenoy $18^{79}$

Chalcedon $82^{232}$

Chalon-sur-Saône $205^{21}$

Châlons-sur-Marne $119,223,239$

Chartres $230^{129}$

Chester $109^{+72}, 152$

Chiusa 117,232

Clairvaux 234147

Clermont-en-Beauvaisis $\quad 18^{79}, 82^{232,235}$, $113^{90}$

Cluny $87^{+260}, 112,114-118,153,156 \mathrm{f}$., 244,249
Compiègne 151,157

Corbeny $62^{140}$

Corbie $28,101^{+39}, 129182,213^{+54}, 214$, $217,221^{+96}, 237^{+167}, 255$

Coucy $1,99,107,128^{+174}, 177^{66}, 202^{6}$

Diedenhofen $85^{249}$

Douai 255

Durham 29

East Anglia $137^{221}$

Echternach 39

England $27,63^{147}, 86^{+252}, 109,117,132$, 137f., 167f., 172, 232f., 248

Essex 123

Exeter $138,145^{258}$

Fécamp $232^{129}$

Flandern $3^{8}, 68^{169}, 88$

Fleury $112,118,131^{189}, 136,145,169$, $172,175,224 \mathrm{f}$.

Florennes 220

Foucarmont $236+156$

Frankreich $15,57^{111}, 63^{147}, 64^{148}$

Gallien 85

Gembloux 236

Grenoble $74 f ., 80^{214}, 146,150,157,162$, 237-239, 240

Griechenland 195

Hastings 27

Irland $134^{202}, 136$

Italien $57^{111}, 87^{259}, 146,177$

Jerusalem $\quad 100^{32}, 196,228^{113}$

Kana 44

Karthago $38^{7}, 85 \mathrm{f}$.

Konstantinopel $68^{+169}, 102^{46}, 199$

Laon $3^{+8}, 13,17^{+76}, 24-26,29 f$., $46 f$., $52^{94}$, $63^{140}, 67,76-78,81^{+224}, 82$, 106f., 119f., $122-125,132,137-139,143,145^{258}$, $146^{261}, 147,151,153^{296}, 154^{303}, 157-159$, $162-165,167$ f., $171,173^{+44}, 174,179^{+80}$, $184,186,188,227,233,249,251$

Lausanne 12

Le Puy 196 
Lessies $80^{218}$

Lihons $82^{233}, 145^{258}$

Limoges 83

Loire 145

London $86^{252}, 115^{99}$

Lothringen 196

Lyon 112,117

Mainz $85^{249}, 86$

Malmesbury 86

Marchiennes $28,32^{164}, 158^{+316}, 211$, $212^{51}, 214^{56}, 221-224,226,235^{147}$

Meaux $80^{212}, 88$

Milvische Brücke 51

Moissac 228114

Mont-Dieu $236^{+159}$

Mont Saint-Michel $232^{129}$

Monte Gargano $211^{44}$

Montecassino 112,116-118, 156f., 168

Mortagne $208^{30}$

Nesle $113^{89}, 145^{258}$

Nogent $1,3,20,24,25^{114}, 26-28,30^{+153}$, $31,32^{164}, 46,78,80^{214}, 81,85,87,99,107$, $109,112,124,127-130,135,144-146$, $156,162-164,167,172,177,179,202^{6}$, 239,247

Nordfrankreich $12,28,57^{111}, 62^{139}, 79$, $81,121,129$

Normandie $117,137,145,236^{156}$

Noyon $124,129^{179}, 130,145,148,157$. 178

Ostanglien $131 \mathrm{f}$.

Ostia 117

Oudenburg 88-91

Ourscamp $89^{269}$

Pamphylische See $49,51,56 \mathrm{f}$.

Paris $32^{163}, 51^{86}, 75,79^{210}, 145,157,237 \mathrm{f}$.

Pavia $232^{129}$

Perche $208^{30}$

Picardie 18, 145

Poitiers $115^{102}$

Pontigny $28,234^{147}$

Porcien 100

Prémontré 24f., $28^{137}$

Ramsey $131,132^{189}$

Reims $3,25,54,76,78-80,81^{221}, 86$, $88^{262.268}, 90,92,101,107,119-122$, $126^{159}, 128^{+174}, 145,153,156,205^{+21}$, $220,225^{102}, 236^{+159 f .}, 239,243,248$
Ripoll 228114

Rom $3^{6}, 117,174,204,212,216,223$, $232^{+129}, 233$

Rotes Meer $44,49,51,53^{100}, 217$

Rouen $82^{232}, 88^{262}, 91,108^{68}, 142$, $208^{+30 f}, 211 \mathrm{f}$.

Saint-Arnoul de Crépy $157^{309}$

Saint-Aubert, Cambrai $213^{+55}, 237^{160}$

Saint-Benoît-sur-Loire $118,212^{+212}$

Saint-Bertin $80^{218}$

Saint-Crépin, Soissons $26,119^{122}, 217^{77}$, $218,220,223-225$

Saint-Denis $27,68,121,133,137 \mathrm{f} ., 145$, $153^{+296}, 157,172,186^{+107}, 212,224 \mathrm{f}$.

Saint-Eloi 129179

Saint-Evroult $137,211^{44}$

Saint-Germer de Fly $18,23107,25^{+116}, 27$, $30^{153}, 53,78,107,109-113^{+896}, 121^{135}$, $142 \mathrm{f} ., 145,147,151-154^{+302}, 157,162$, $164,167^{+22}, 175 \mathrm{f} ., 177^{66}, 247,249$

Saint-Ghislain 238

Saint-Jean-d'Angély $\quad 68^{+169}, 199$

Saint-Just-en-Chaussée $140,170^{40}, 177$

Saint-Lucien, Beauvais $\quad 80^{217}$

Saint-Maclou $62^{140}$

Saint-Martin de Tournai $129^{179}, 130,234$, 237,270

Saint-Martin-du-Bosc $109^{73}$

Saint-Maur-des-Fossés $\quad 129^{182}, 154^{302}$

Saint-Médard de Soissons $8,25^{+119}, 28$, $31,38,40,47,51,62^{139}, 67,70^{+175}, 80^{212}$, $88,93,127^{+165}, 143 \mathrm{f}$., $152,158^{+310}, 174$, $180,183^{+99}, 184,201-213^{+53 f}, 215-218^{78}$, $220-225^{+102}, 230^{129}, 246,251 \mathrm{f} ., 255$

Saint-Nicaise, Reims $25,153,236^{159}$

Saint-Nicolas-aux-Bois $\quad 24,209^{32}$

Saint-Omer 77

Saint-Pierre, Beauvais $170^{40}$

Saint-Quentin, Beauvais $14,60,62,76$, $80^{217}, 113^{89}, 115,120,125,148$

Saint-Rémi, Reims $\quad 47^{61}, 101,128,205^{21}$, $236^{159}$

Saint-Riquier 72

Saint-Sépulchre, Cambrai $102^{46}, 129^{181}$, $213^{55}, 234^{+145}, 237,269 \mathrm{f}$.

Saint-Symphorien, Beauvais $25,113^{89}$

Saint-Thierry, Reims 25, 225 102,236

Saint-Vincent, Laon $24,234^{145}$

Saints-Crépin-et-Crépinien, Soissons s. Saint-Crépin, Soissons

Salisbury 123

Santiago de Compostela 113,146 
Semur $113,115^{101}$

Sens $88^{262}, 91$

Signy $236,237^{160}, 243$

Soissons $24-29,43,57^{116}, 59 f$ f, 62, 76, 80 212 f. $81^{221}, 84^{+244}, 88,89^{271}, 91,119^{122}$, $120,145,152,158,164,182,194^{147}, 202^{6}$, $203,205,207,210,212,216-218,220$, $222-224,225^{102}, 227,252,269$

Soissonnais 164

St. Emmeram 186

St. Saba 117,233

Straßburg 12

Südengland 145

Sussex $109^{73}$
Tal Josaphat $228^{113}$

Thérouanne $121^{135}$

Tongerloo 238

Tours $157^{309}$

Touraine $145^{258}$

Tournai 88,90

Trier $91,208^{30}$

Troyes $80^{213}$

Vexin 145,171

Viviers 74f., 157, 238f.

Winchester $138,145^{258}, 157,179$

Westminster 86 

